\title{
Feasibility of Applying the Extended ICF Core Set for Stroke to Clinical Settings in Rehabilitation: A Preliminary Study
}

\author{
Kyu Yong Han, MD, Hyo Jong Kim, MD, Heui Je Bang, MD \\ Department of Rehabilitation Medicine, Chungbuk National University College of Medicine, Cheongju, Korea
}

\begin{abstract}
Objective To evaluate the potential feasibility of application of the extended International Classification of Functioning, Disability and Health (ICF) Core Set for stroke.

Methods We retrospectively reviewed the medical records of 40 stroke outpatients ( $>6$ months after onset) admitted to the Department of Rehabilitation Medicine for comprehensive rehabilitation. Clinical information of the patients were respectively evaluated to link to the 166 second-level categories of the extended ICF Core Set for stroke.

Results Clinical information could be linked to 111 different ICF categories, 58 categories of the body functions component, eight categories of the body structures component, 38 categories of the activities and participation component, and seven categories of the environmental factors component.

Conclusion The body functions component might be feasible for application of the extended ICF Core Set for stroke to clinical settings. The activities and participation component and environmental factors component may not be directly applied to clinical settings without additional evaluation tools including interview and questionnaire.
\end{abstract}

Keywords International Classification of Functioning, Disability and Health (ICF), Functioning, Disability, Health, Stroke

\section{INTRODUCTION}

Stroke rehabilitation includes a professional team in-

Received August 5, 2014; Accepted September 8, 2014

Corresponding author: Heui Je Bang

Department of Rehabilitation Medicine, Chungbuk National University College of Medicine, 776 1(il)sunhwan-ro, Heungdeok-gu, Cheongju 361711 , Korea

Tel: +82-43-269-6228, Fax: +82-43-269-6227, E-mail: bang@chungbuk.ac.kr

(c) This is an open-access article distributed under the terms of the Creative Commons Attribution Non-Commercial License (http://creativecommons. org/licenses/by-nc/3.0) which permits unrestricted noncommercial use, distribution, and reproduction in any medium, provided the original work is properly cited.

Copyright () 2015 by Korean Academy of Rehabilitation Medicine cluding a physical therapist, occupational therapist, speech therapist, clinical psychologist, nurse, social worker, and physiatrist. This team requires multidisciplinary medical services in terms of various sequelae. Challenges in stroke patients include complexities, such as cognitive impairment, language and communication disorders, motor and sensory impairment, dysphagia, spasticity, hemispatial neglect, shoulder pain, depression, and voiding dysfunction [1-3].

Such a team approach is based on efficient communication between team members, and in particular, the use of common language for research, comparison and 
analysis of many organizations and countries [2]. To address this goal the World Health Organization developed the International Classification of Functioning, Disability and Health (ICF) in 2001. The aim of the ICF is to help with promotive communication among policy-makers, health-related specialists and the general public by providing the scientific basis and comparison of health and health-related data between countries to further an understanding and study of health, health-related states, outcomes and determinants [4]. The ICF provides quality communication and clarifies the team roles between members of the rehabilitation team in stroke rehabilitation [5]. In addition, ICF has the potential value to establish treatment plans for patients, which is applied as a useful framework for measuring the disability and functions in stroke-related clinical research [6-8].

The original version of ICF enabled evaluation of the overall health and its relevant fields. However, the process describing a person's functioning and health requires at least 1 hour to due to the 1,424 categories in four components (body functions, body structures, activities and participation, and environmental factors). So, the original form of ICF proved impractical [2] and spurred the development of the comprehensive ICF Core Set for stroke with 130 categories and the brief ICF Core Set for stroke with 18 categories for an easier clinical application of the typical spectrum of problems in patients with stroke [9]. An extended ICF Core Set for stroke was subsequently suggested. This core set added 36 categories to the comprehensive ICF Core Set for stroke to enable its use in acute and early post-acute phases [10-12].

Studies using the ICF Core Set for stroke have mainly identified the linking frequency of each category of the ICF Core Set for stroke and its validity through understanding of factors including the impairment and activity limitation of stroke patients based on interviews, questionnaires, physical examinations, and medical records [13-16]. Other studies also reported that various examinations and functional assessment tools used in rehabilitation studies are linked to any category of the original version of ICF $[7,17,18]$. The ICF has to be oriented so there is an easier and more convenient clinical application for the international statistical standards related to health and the systemic classification and comparison through the standards. However, except for some clinical researchers and doctors, treat patients and prepare medi- cal records regardless of the concept of the ICF in Korea after 13 years. Few studies have been conducted, even though the linkage between the current medical records of stroke patients and the categories of the extended ICF Core Set for stroke is very significant when it comes to judging the potential for an easier clinical application of ICF, as medical records include all data for a patient without exception in the clinical field.

This preliminary study retrospectively reviewed the medical records for stroke patients in two hospitals to identify the extent of the linkage with the categories of the extended ICF Core Set for stroke, and evaluated the potential feasibility of the application of the extended ICF Core Set for stroke to rehabilitation-relevant clinical settings.

\section{MATERIALS AND METHODS}

\section{Subjects}

This study was approved by the Institutional Review Board of Chungbuk National University Hospital. We reviewed the medical records of the stroke outpatients at least 6 months after the onset of a stroke that had been admitted to the Department of Rehabilitation Medicine for comprehensive rehabilitation at Chungbuk National University Hospital and Cheongju Medical Center, Cheongju, Korea. The medical records were all of the relevant records from the Departments of stroke-related Neurology, Neurosurgery, Cardiology, Psychology, and Rehabilitation Medicine. Forty patients, 20 from each hospital, were selected by the latest onset as of the study period.

The 40 patients comprised 19 males $(47.5 \%)$ and 21 females $(52.5 \%)$ with a mean age of $63.5 \pm 12.6$ years. There were 21 right hemiplegic patients $(52.5 \%)$ and 19 left hemiplegic patients (47.5\%). Ten patients $(25.0 \%)$ had a hemorrhagic stroke and 30 patients $(75.0 \%)$ had an ischemic stroke. Their average duration of illness after stroke was $13.0 \pm 4.4$ months and the length of stay was $46.5 \pm 21.0$ days.

\section{Linkage between medical records and ICF categories}

The retrospective review of all the stroke-related hospitalization and outpatient clinic medical records was done. However, the medical records that were created before the stroke were excluded. A physiatrist who had ICF 
experience reviewed all the various assessment results of the Korean version of Modified Barthel Index (K-MBI), Korean Mini-Mental Status Examination (K-MMSE), Korean version of Western Aphasia Battery (K-WAB), Korean version of Hamilton Depression Rating Scale (K-HDRS), and Korean-Wechsler Adult Intelligence Scale (K-WAIS), as well as the admission, referral, collaborative treatment, nursing, outpatient and the rehabilitation-related records. In addition, results of imaging studies and laboratory tests were reviewed. Then, each item of the medical records was investigated to establish the extent of linkage to the second-level categories of the extended ICF
Core Set for stroke. If the content of each medical record were linked to one or more of the proper third or fourth categories subclassified in the second level of ICF, it was regarded as being linked to the second level of the upper classification for details in the medical record regardless of the normal or abnormal status.

The extended ICF Core Set for stroke consisted of 166 categories that belonged to the second level of the ICF. It includes 59, 11, 59, and 37 categories in the body functions component, body structures component, activities and participation component, and environmental factors component, respectively. The linkage between each item

Table 1. Examples of the linking process

\section{ICF category}

\begin{tabular}{ll} 
Technical measures & \\
Electrocardiography & b410 Heart functions \\
\hline Echocardiography & b410 Heart functions \\
\hline Pulse wave velocity and ankle brachial index & s410 Structure of cardiovascular system \\
\hline Chest X-ray or chest computed tomography & b415 Blood vessel functions \\
Pulmonary function test & s430 Structure of respiratory structure \\
Electrolyte & b440 Respiration functions \\
\hline Complete blood cell count & b545 Water, mineral and electrolyte balance functions \\
Blood glucose & b430 Haematological system functions \\
\hline White blood cell count & b540 General metabolic functions \\
Clinical measures & b435 Immunological system functions \\
\hline Respiration rate & \\
\hline Blood pressure & b440 Respiration functions \\
Voluntary coughing & b420 Blood pressure functions \\
Body weight & b450 Additional respiratory functions \\
Body temperature & b530 Weight maintenance functions \\
Manual muscle test & b550 Thermoregulatory functions \\
\hline Deep tendon reflex & b730 Muscle power functions \\
Assessment tools & b750 Muscle reflex functions \\
\hline Modified Barthel Index & d420 Transferring oneself \\
\hline & d450 Walking \\
\hline & d455 Moving around \\
\hline & d465 Moving around using equipment \\
\hline & d510 Washing oneself \\
\hline & d520 Caring for body parts \\
\hline & d530 Toileting \\
\hline & d550 Eating \\
\hline
\end{tabular}

ICF, International Classification of Functioning, Disability and Health. 
of the medical record and the proper ICF category was referred to as ICF application manual for users, and some methods have been used in many studies [19-23].

The linkage between each item of the medical records and the third or fourth level categories of ICF was mainly decided by understanding the purpose of the details of the medical records. For example, the item electrolyte in the medical records, which checks for an electrolyte balance/imbalance in the body, can be linked to 'Electrolyte balance (b5452)' of the third level category in body functions. As a result, it was considered to be linked to 'Water, mineral and electrolyte balance functions (b545)' of the second level category of the upper classification in body functions (Table 1). If some medical records were not clearly linked to the ICF categories, the physiatrist would discuss the case with relevant professionals in specific fields, such as physician, physical therapist, occupational therapist, speech therapist, and psychologist to determine whether it was linked or not.

\section{Analysis}

Linkage analysis results between the details of the medical records and the ICF categories were analyzed using descriptive statistics. In 166 categories included in the extended ICF Core Set for stroke, we investigated the second-level categories linked to one or more medical records, and the frequency for each second-level category. They were described at $76 \%-100 \%, 51 \%-75 \%, 26 \%-50 \%$, and $0 \%-25 \%$ from higher to lower frequency for each component. The average number and percentage of the categories linked from all patients were investigated, and were described for each component and each hospital.

\section{RESULTS}

\section{Body functions}

Except for alternative vocalization functions (b340), 58 out of 59 categories included in the extended ICF Core Set for stroke were linked to the medical record of at least one patient. Fifty ICF categories were linked to $76 \%$ or more medical records from 40 patients. Temperament and personality functions (b126, 15\%) and alternative vocalization functions (b340, $0 \%$ ) were linked by $25 \%$ or lower frequency (Table 2).

\section{Body structures}

Eight out of a total of 11 categories of the body structures were evaluated. Five categories-structure of brain (s110, 100\%), structure of cardiovascular system (s410, $100 \%)$, structure of respiratory structure (s430, 100\%), structure of shoulder region (s720, 100\%), and structure of areas of skin (s810, 100\%)-were evaluated in all of the medical records of patients. Three unevaluated categories were spinal cord and related structure (s120, $0 \%)$, structure of meninges (s130,0\%), and structure of upper extremity (s730, 0\%) (Table 3). In these categories relevant data could not be found in the medical records because the patient did not have any abnormal findings so there was no need to do additional examinations and further evaluation.

Table 2. Evaluated ICF code of the body functions component included in the extended ICF Core Set for stroke, listed in order of frequency

\begin{tabular}{ccc}
\hline $\begin{array}{c}\text { Frequency } \\
(\%)\end{array}$ & \multicolumn{1}{c}{ ICF code (\%) } & $\begin{array}{c}\text { Total } \\
(\mathbf{n}=\mathbf{5 9})\end{array}$ \\
\hline $76-100$ & b110 (100), b114 (100), b130 (100), b134 (100), b152 (100), b156 (100), b167 (100), b176 (100), & 50 \\
& b180 (100), b210 (100), b215 (100), b230 (100), b235 (100), b240 (100), b260 (100), b265 (100), \\
& b270 (100), b280 (100), b310 (100), b320 (100), b410 (100), b420 (100), b430 (100), b435 (100), \\
& b440 (100), b510 (100), b515 (100), b525 (100), b530 (100), b535 (100), b540 (100), b545 (100), \\
& b550 (100), b620 (100), b630 (100), b710 (100), b715 (100), b730 (100), b735 (100), b740 (100), \\
& b750 (100), b755 (100), b760 (100), b770 (100), b810 (100), b117 (92.5), b140 (92.5), b144 \\
& (92.5), b164 (92.5), b172 (92.5) & 3 \\
\hline $51-75$ & b415 (75.0), b330 (72.5), b147 (62.5) & 4 \\
\hline $26-50$ & b160 (50.0), b455 (50.0), b640 (50.0), b450 (37.5) & 2 \\
\hline $0-25$ & b126 (15.0), b340 (0) & \\
\hline
\end{tabular}

ICF, International Classification of Functioning, Disability and Health. 


\section{Activities and participation}

Thirty-eight of 59 activities and participation categories were evaluated. Twenty-eight were linked to the medical records of $76 \%$ or more patients. Twenty-one unevaluated categories were carrying out daily routine (d230, 0\%), using communication devices and techniques (d360, 0\%), using transportation (d470, 0\%), driving (d475, 0\%), drinking (d560, 0\%), looking after one's health (d570, 0\%), acquisition of goods and services (d620,0\%), preparing meals (d630,0\%), doing housework (d640,0\%), basic interpersonal interactions (d710, 0\%), informal social relationships (d750, 0\%), intimate relationships (d770, 0\%), acquiring, keeping and terminating a job (d845, 0\%), remunerative employment (d850, 0\%), non-remunerative employment (d855, 0\%), basic economic transactions (d860,0\%), economic self-sufficiency (d870,0\%), community life (d910, 0\%), recreation and leisure (d920, 0\%), religion and spirituality (d930,0\%), and human rights (d940, 0\%) (Table 4).

\section{Environmental factors}

Seven out of 37 categories in the environmental fac- tors-products or substances for personal consumption (e110, 100\%), immediate family (e310, 72.5\%), products and technology for personal indoor and outdoor mobility and transportation (e120,60\%), personal care providers and personal assistants (e340,37.5\%), social security services, systems and policies (e570,17.5\%), products and technology for personal use in daily living (e115, 12.5\%), and individual attitudes of immediate family members (e410, 7.5\%) - were evaluated. The others were not (Table $5)$.

\section{Comparison between hospitals}

In the study sample, the average number of evaluated categories was $90.9 \pm 6.0(54.7 \% \pm 3.6 \%)$ for each patient. Body functions, body structures, activities and participation, and environmental factors categories comprised $53.8 \pm 3.4(91.1 \% \pm 5.8 \%)$ of 59 categories, $5.2 \pm 0.4$ $(47.1 \% \pm 3.5 \%)$ of 11 categories, $28.9 \pm 2.6(48.9 \% \pm 4.4 \%)$ of 59 categories and $3.1 \pm 1.1(8.3 \% \pm 3.1 \%)$ of 37 categories, respectively.

For each general and university hospital, the average number of the evaluated categories was $86.4 \pm 5.3$

Table 3. Evaluated ICF code of the body structures component included in the extended ICF Core Set for stroke, listed in order of frequency

\begin{tabular}{ccc}
\hline Frequency (\%) & ICF code (\%) & Total (n=11) \\
\hline $76-100$ & s110 (100), s410 (100), s430 (100), s720 (100), s810 (100) & 5 \\
\hline $51-75$ & - & 0 \\
$26-50$ & - & 0 \\
$0-25$ & s750 (10.0), s530 (5.0), s710 (2.5), s120 (0), s130 (0), s730 (0) \\
\hline
\end{tabular}

ICF, International Classification of Functioning, Disability and Health.

Table 4. Evaluated ICF code of the component activities and participation included in the extended ICF Core Set for stroke, listed in order of frequency

\begin{tabular}{|c|c|c|}
\hline Frequency (\%) & ICF code (\%) & Total $(n=59)$ \\
\hline $76-100$ & $\begin{array}{l}\mathrm{d} 110 \text { (100), d115 (100), d120 (100), d210 (100), d310 (100), d330 (100), d350 (100), } \\
\text { d410 (100), d415 (100), d420 (100), d430 (100), d440 (100), d445 (100), d450 (100), } \\
\text { d455 (100), d465 (100), d510 (100), d520 (100), d530 (100), d540 (100), d550 (100), } \\
\text { d130 (92.5), d160 (92.5), d166 (92.5), d170 (92.5), d172 (92.5), d177 (92.5), d325 (87.5) }\end{array}$ & 28 \\
\hline $51-75$ & - & 0 \\
\hline $26-50$ & $\mathrm{~d} 460(40.0), \mathrm{d} 760(37.5)$ & 2 \\
\hline $0-25$ & $\begin{array}{l}\mathrm{d} 155(17.5), \mathrm{d} 315(10.0), \mathrm{d} 135(7.5), \mathrm{d} 175(7.5), \mathrm{d} 240(7.5), \mathrm{d} 335(7.5), \mathrm{d} 220(5.0), \\
\text { d345 (5.0), d230(0), d360 (0), d470(0), d475(0), d560 (0), d570 (0), d620(0), d630(0), } \\
\text { d640 (0), d710 (0), d750 (0), d770 (0), d845 (0), d850 (0), d855 (0), d860 (0), d870 (0), } \\
\text { d910 (0), d920 (0), d930 (0), d940 (0) }\end{array}$ & 29 \\
\hline
\end{tabular}

ICF, International Classification of Functioning, Disability and Health. 
Table 5. Evaluated ICF code of the component environmental factors included in the extended ICF Core Set for stroke, listed in order of frequency

\begin{tabular}{clc}
\hline Frequency (\%) & \multicolumn{1}{c}{ ICF code (\%) } & Total (n=37) \\
\hline $76-100$ & e110 (100) & 1 \\
$51-75$ & e310 (72.5), e120 (60.0) & 1 \\
$26-50$ & e340 (37.5) & 33 \\
$0-25$ & e570 (17.5), e115 (12.5), e410 (7.5), e125 (0), e135 (0), e150 (0), e155 (0), e165 (0), \\
& e210 (0), e240 (0), e250 (0), e315 (0), e320 (0), e325 (0), e355 (0), e360 (0), e415 (0), \\
& e420 (0), e425 (0), e440 (0), e450 (0), e455 (0), e460 (0), e465 (0), e515 (0), e525 (0), \\
& e535 (0), e540 (0), e550 (0), e555 (0), e575 (0), e580 (0), e590 (0) \\
\hline
\end{tabular}

ICF, International Classification of Functioning, Disability and Health.

$(52.1 \% \pm 3.2 \%)$ and $95.4 \pm 1.9(57.4 \% \pm 1.2 \%)$, respectively. It was slightly higher in the university hospital than the general hospital. The general hospital and university hospital were $51.0 \pm 2.6(86.3 \% \pm 4.4 \%)$ and $56.6 \pm 0.7$ $(95.9 \% \pm 1.2 \%)$ in 59 categories of the body functions, $5.1 \pm 0.3(46.4 \% \pm 2.8 \%)$ and $5.3 \pm 0.4(47.7 \% \pm 4.0 \%)$ in 11 categories of the body structure, $27.9 \pm 3.0(47.3 \% \pm 5.1 \%)$ and $29.9 \pm 1.6(50.6 \% \pm 2.7 \%)$ in 59 categories of activities and participation, and $2.5 \pm 1.1(6.8 \% \pm 3.1 \%)$ and $3.7 \pm 0.8$ $(9.9 \% \pm 2.2 \%)$ in 37 categories of the environmental factors for each component.

\section{DISCUSSION}

The ICF has to be easily applied to the clinic and health fields too far from its original definition and developmental purposes to become the common language of the professionals and the public in their relevant fields, as well as being utilized as one of the international standard health-related statistics. The comprehensive ICF Core Set and brief ICF Core Set have been developed to use for each specific condition because the full version of the ICF consists of 1,424 categories in four components. Many researchers and professionals from the global community have studied and utilized the extended ICF Core Set for stroke in the clinic and health fields. However, the international standards have not yet been prepared. This study compared 166 categories of the extended ICF Core Set for stroke with the medical records to evaluate the potential feasibility of the application of the ICF for stroke. The average number of evaluated categories was $90.9 \pm 6.0$ $(54.7 \% \pm 3.6 \%)$ in a total of 40 patients, representing about half of the extended ICF Core Set for stroke. A total of 111 different ICF categories were evaluated, 58 categories of the body functions component, eight categories of the body structures component, 38 categories of the activities and participation component, and seven categories of the environmental factors component.

In the body functions, 58 categories, except alternative vocalization functions (b340,0\%), from a total of 59 were evaluated. Five of 49 categories with $76 \%$ or higher frequency-intellectual functions (b117, 92.5\%), attention functions (b140, 92.5\%), memory functions (b144, 92.5\%), higher-level cognitive functions (b164, 92.5\%) and calculation functions (b172, 92.5\%) - were evaluated by the routine examination. However, some medical records were omitted without clear reasons. Therefore they might be considered to be evaluated. Thought functions (b160, 50\%) from four categories with $26 \%-50 \%$ frequency were evaluated, similar to a prior study [23], using KWAIS. Two categories, exercise tolerance functions ( $b 455$, $50 \%$ ) and sexual functions (b640,50\%), were evaluated by K-HDRS. These two tests were done only in the university hospital and the above three categories displayed $50 \%$ frequency. Additional respiratory functions (b450, $37.5 \%$ ) were evaluated by videofluoroscopic swallowing study or Gugging Swallowing Screen applied to some patients with dysphagia and displayed $37.5 \%$ frequency. Temperament and personality functions (b126, 15\%) having two categories with $\leq 25 \%$ frequency were evaluated by the medical records written by psychiatrists. Alternative vocalization functions (b340,0\%) are defined in the ICF as other manners of vocalization like "functions of the production of notes and range of sounds" [4] and the category was considered to be unevaluated because both hospitals did not check the notes and range of sounds. However, if the melodic intonation therapy [24] is applied to the patient with aphasia, this category can be consid- 
ered to be evaluated in some cases. The majority of the 59 categories of body functions included in the extended ICF Core Set for stroke which represent various problems shown in the stroke were evaluated in the current clinical area.

In the body structures, eight of a total of 11 categories were evaluated in one or more medical records of the patients. Structure of brain (s110, 100\%) in five categories with $100 \%$ frequency was evaluated in all stroke patients. The other four categories with $100 \%$ frequency-structure of cardiovascular system (s410,100\%), structure of respiratory structure (s430,100\%), structure of shoulder region (s720, 100\%), and structure of areas of skin (s810, 100\%) - are highly related to post-stroke complications $[25,26]$. Structure of shoulder region $(s 720,100 \%)$ is related with stability of joint functions $(b 715,100 \%)$ in the body functions. They displayed $100 \%$ frequency because all stroke patients are checked by the imaging study or fingerbreadth palpation method for hemiplegic shoulder $[26,27]$. Structure of lower extremity (s750, 10\%), structure of stomach (s530,5\%), and structure of head and neck region (s710, 2.5\%) from six categories with $\leq 25 \%$ frequency were recorded only if the organ for each patient had a problem. If not, they were considered to be unevaluated. Therefore, spinal cord and related structures (s120,0\%), structures of meninges (s130,0\%), and structures of upper extremity (s730, 0\%) displayed $0 \%$ frequency. Most of the medical records are practically described in case of the body structures with problems or expected complications. Therefore, all categories of body structures could be considered to be $100 \%$ evaluated, although three categories were not evaluated in this study.

Thirty-eight of 59 categories in activities and participation were evaluated in one or more medical records. The others were not. Eight categories-transferring oneself (d420, 100\%), walking (d450, 100\%), moving around (d455, 100\%), washing oneself (d510, 100\%), caring for body parts (d520, 100\%), toileting (d530, 100\%), dressing (d540, 100\%), and eating (d550, 100\%)-from 21 categories with $100 \%$ frequency were checked by K-MBI [21].

Six categories with $92.5 \%$ frequency-copying (d130, 92.5\%), focusing attention (d160, 92.5\%), reading (d166, 92.5\%), writing (d170, 92.5\%), calculating (d172, 92.5\%), and making decisions (d177, 92.5\%)—were due to some patients with omitted medical records. They were considered to be $100 \%$ evaluated. Eight categories-acquir- ing skills (d155, 17.5\%), communicating with - receiving - non-verbal messages (d315, 10\%), rehearsing (d135, 7.5\%), solving problems (d175, 7.5\%), handling stress and other psychological demands (d240, 7.5\%), producing non-verbal messages (d335, 7.5\%), undertaking multiple tasks (d220,5\%), and writing messages (d345, 5\%)—from 29 categories with $\leq 25 \%$ frequency were due to categories not related to the routine examinations in the clinical practice. Fifteen categories, which were included in chapter 6 (domestic life), chapter 8 (major life areas), chapter 9 (community, social and civic life), and chapter 4 (mobility in the activities and participation), from 21 unevaluated categories were thought to be the categories related to the social activities after leaving a hospital. They were not found in the medical records. Drinking (d560, 0\%) is a very important category for the stroke patients, but we could not find any description of it. Three categories-solving problems (d175, 0\%), drinking (d560, $0 \%)$, and basic interpersonal interaction (d710, 0\%)might be evaluated as previously [28] using the Functional Independence Measure (FIM).

Seven of 37 categories about environmental factors were evaluated in one or more medical records. The other 30 categories were considered to be unevaluated. Products or substances for personal consumption (e110, $100 \%$ ) of seven evaluated categories displayed $100 \%$ frequency because some descriptions about the response of the drug therapy were found in all of the medical records. Immediate family (e310, 72.5\%) and products and technology for personal indoor and outdoor mobility and transportation (e120,60\%) displayed 51\%-75\% frequency, and personal care providers and personal assistants (e340, 37.5\%) and products and technology for personal use in daily living $(e 115,12.5 \%)$ displayed $\leq 50 \%$ frequency were considered to be evaluated if we could find some description in the medical records about help provided by caregivers including family or the transfer activity using assistive devices. Social security services, systems and policies (e570, 17.5\%) was considered to be evaluated if we could find some descriptions about disability assessment for national registration in the medical records. This study is similar to other studies in that the categories about the environmental factors were rarely linked to the assessment tools used in the rehabilitation research $[17,18]$.

Concerning the extended ICF Core Set for stroke, stud- 
ies conducted outside of Korea reported the validation from the perspective of disease course, as well as patient's gender and health professionals [15,29-31]. Two studies used the categories of the comprehensive or brief ICF core set for stroke in Korea [32,33]. However, we could not find any study using the extended ICF Core Set for stroke in the literature.

There were some limitations. First, this study involved only local hospitals and a small number of subjects. Second, we did not apply the qualifier for each category regardless of the aim of ICF. Third, the details of some medical records were subjectively linked to the ICF categories without clear evidence, which is a feature of studies using the ICF. In studies of ICF that used FIM, each researcher linked the same evaluation tool to the different categories. Kim et al. [21] linked 'Regulating urination (d5300)' and 'Regulating defecation (d5301)' in activities and participation to 'Bladder management and Bowel management' of FIM. Kohler et al. [28] linked 'Urination functions (b620)' and 'Defecation functions (b525)' of body functions to 'Bladder management and Bowel management' of FIM. De Vriendt et al. [34] linked MMSE to five ICF categories-orientation functions (b114), attention functions (b140), memory functions (b144), mental functions of language (b167), and undertaking a single task (d210). Campos et al. [22] linked MMSE to 21 ICF categories.

In conclusion, in the current clinical setting, 111 of 166 categories in the extended ICF Core Set for stroke were evaluated by reviewing the medical records of 40 stroke patients. In the body functions, 58 of 59 categories were considered to be evaluated. The body functions component might be feasible for application of the extended ICF Core Set for stroke to clinical settings. In activities and participation and the environmental factors components, 38 of 59 and seven of 37 categories, respectively, were considered to be evaluated separately. The component activities and participation and environmental factors categories cannot be directly applied to clinical settings without additional evaluation tools including interview and questionnaire.

\section{CONFLICT OF INTEREST}

No potential conflict of interest relevant to this article was reported.

\section{ACKNOWLEDGMENTS}

This work was supported by a research grant from Chungbuk National University in 2013.

\section{REFERENCES}

1. Harvey RL, Roth EJ, Yu DT, Celnik P. Stroke syndromes. In: Braddom RL, editor. Physical medicine and rehabilitation. 4th ed. Philadelphia: Saunders; 2010. p. 1177-222.

2. Stucki G, Ewert T, Cieza A. Value and application of the ICF in rehabilitation medicine. Disabil Rehabil 2002;24:932-8.

3. Ustun B, Chatterji S, Kostanjsek N. Comments from WHO for the Journal of Rehabilitation Medicine Special Supplement on ICF Core Sets. J Rehabil Med 2004;(44 Suppl):7-8.

4. World Health Organization. International classification of functioning, disability and health: ICF. Geneva: World Health Organization; 2001.

5. Tempest S, McIntyre A. Using the ICF to clarify team roles and demonstrate clinical reasoning in stroke rehabilitation. Disabil Rehabil 2006;28:663-7.

6. Rentsch HP, Bucher P, Dommen Nyffeler I, Wolf C, Hefti H, Fluri E, et al. The implementation of the 'International Classification of Functioning, Disability and Health' (ICF) in daily practice of neurorehabilitation: an interdisciplinary project at the Kantonsspital of Lucerne, Switzerland. Disabil Rehabil 2003;25:41121.

7. Geyh S, Cieza A, Kollerits B, Grimby G, Stucki G. Content comparison of health-related quality of life measures used in stroke based on the international classification of functioning, disability and health (ICF): a systematic review. Qual Life Res 2007;16:833-51.

8. Salter KL, Foley NC, Jutai JW, Teasell RW. Assessment of participation outcomes in randomized controlled trials of stroke rehabilitation interventions. Int J Rehabil Res 2007;30:339-42.

9. Geyh S, Cieza A, Schouten J, Dickson H, Frommelt P, Omar Z, et al. ICF Core Sets for stroke. J Rehabil Med 2004;(44 Suppl):135-41.

10. Grill E, Lipp B, Boldt C, Stucki G, Koenig E. Identification of relevant ICF categories by patients with neurological conditions in early post-acute rehabilitation 
facilities. Disabil Rehabil 2005;27:459-65.

11. Stier-Jarmer M, Grill E, Ewert T, Bartholomeyczik S, Finger M, Mokrusch T, et al. ICF Core Set for patients with neurological conditions in early post-acute rehabilitation facilities. Disabil Rehabil 2005;27:389-95.

12. Ewert T, Grill E, Bartholomeyczik S, Finger M, Mokrusch T, Kostanjsek N, et al. ICF Core Set for patients with neurological conditions in the acute hospital. Disabil Rehabil 2005;27:367-73.

13. Riberto M, Lopes KA, Chiappetta LM, Lourencao MI, Battistella LR. The use of the comprehensive International Classification of Functioning, Disability and Health core set for stroke for chronic outpatients in three Brazilian rehabilitation facilities. Disabil Rehabil 2013;35:367-74.

14. Saltychev M, Tarvonen-Schroder S, Eskola M, Laimi K. Selecting an optimal abbreviated ICF set for clinical practice among rehabilitants with subacute stroke: retrospective analysis of patient records. Int J Rehabil Res 2013;36:172-7.

15. Glassel A, Coenen M, Kollerits B, Cieza A. Content validation of the International Classification of Functioning, Disability and Health core set for stroke from gender perspective using a qualitative approach. Eur J Phys Rehabil Med 2014;50:285-99.

16. Wang P, Li H, Guo Y, Xie Y, Ge R, Qiu Z. The feasibility and validity of the comprehensive ICF core set for stroke in Chinese clinical settings. Clin Rehabil 2014;28:159-71.

17. Scheuringer M, Grill E, Boldt C, Mittrach R, Mullner $\mathrm{P}$, Stucki G. Systematic review of measures and their concepts used in published studies focusing on rehabilitation in the acute hospital and in early post-acute rehabilitation facilities. Disabil Rehabil 2005;27:41929.

18. Geyh S, Kurt T, Brockow T, Cieza A, Ewert T, Omar Z, et al. Identifying the concepts contained in outcome measures of clinical trials on stroke using the International Classification of Functioning, Disability and Health as a reference. J Rehabil Med 2004;(44 Suppl):56-62.

19. Statistics Korea. ICF application manual for users. 1st ed. Seoul: Statistics Korea; 2010. p. 1-85.

20. Cieza A, Geyh S, Chatterji S, Kostanjsek N, Ustun B, Stucki G. ICF linking rules: an update based on lessons learned. J Rehabil Med 2005;37:212-8.
21. Kim W, Ahn MR, Kim ES, Lee JE, Lee MJ, Kim MS. Function and environmental factors analysis using ICF (International Classification of Functioning, Disability and Health) for people with disabilities. J Korean Acad Rehabil Med 2008;32:100-5.

22. Campos TF, Rodrigues CA, Farias IM, Ribeiro TS, Melo LP. Comparison of instruments for sleep, cognition and function evaluation in stroke patients according to the international classification of functioning, disability and health (ICF). Rev Bras Fisioter 2012;16:23-9.

23. Laxe S, Tschiesner U, Zasler N, Lopez-Blazquez R, Tormos JM, Bernabeu M. What domains of the International Classification of Functioning, Disability and Health are covered by the most commonly used measurement instruments in traumatic brain injury research? Clin Neurol Neurosurg 2012;114:645-50.

24. Sparks RW, Holland AL. Method: melodic intonation therapy for aphasia. J Speech Hear Disord 1976; 41:287-97.

25. Blecic S, Bogousslavsky J. General management of patients with ischaemic stroke: clinical features and epidemiology. Curr Opin Neurol 1995;8:30-7.

26. Wanklyn P, Forster A, Young J. Hemiplegic shoulder pain (HSP): natural history and investigation of associated features. Disabil Rehabil 1996;18:497-501.

27. Najenson T, Pikielny SS. Malalignment of the glenohumeral joint following hemiplegia: a review of 500 cases. Ann Phys Med 1965;8:96-9.

28. Kohler F, Connolly C, Sakaria A, Stendara K, Buhagiar M, Mojaddidi M. Can the ICF be used as a rehabilitation outcome measure? A study looking at the inter- and intra-rater reliability of ICF categories derived from an ADL assessment tool. J Rehabil Med 2013;45:881-7.

29. Glassel A, Kirchberger I, Kollerits B, Amann E, Cieza A. Content validity of the Extended ICF Core Set for stroke: an international Delphi survey of physical therapists. Phys Ther 2011;91:1211-22.

30. Lemberg I, Kirchberger I, Stucki G, Cieza A. The ICF Core Set for stroke from the perspective of physicians: a worldwide validation study using the Delphi technique. Eur J Phys Rehabil Med 2010;46:377-88.

31. Alguren B, Lundgren-Nilsson A, Sunnerhagen KS. Functioning of stroke survivors: a validation of the ICF core set for stroke in Sweden. Disabil Rehabil 2010;32:551-9. 
32. Park MJ, Kang J. ICF Core Sets for measuring functional status of acute stroke patients. J Muscle Joint Health 2012;19:107-19.

33. Kang J, Gong J, Park MJ, Lee YW, Kim Y. Assessing the functional status of acute stroke patients using ICF Core Set-short form. J Muscle Joint Health 2013;20:22-
30.

34. De Vriendt P, Gorus E, Bautmans I, Mets T. Conversion of the Mini-Mental State Examination to the International Classification of Functioning, Disability and Health terminology and scoring system. Gerontology 2012;58:112-9. 